\title{
Quantitative Determination of a Nonpeptide Antithrombotic in Dog Plasma by Microbore High-Performance Liquid Chromatography-Tandem Mass Spectrometry Utilizing Pneumatically Assisted Electrospray Ionization
}

\author{
Richard C. Simpson* \\ Department of Drug Metabolism and Pharmacokinetics, SmithKline Beecham Pharmaceuticals, King of \\ Prussia, Pennsylvania, USA
}

\begin{abstract}
A method has been developed and is described for the quantitative determination of a nonpeptide antithrombotic in dog plasma. The assay employs reversed phase microbore high-performance liquid chromatography in conjunction with tandem mass spectrometry utilizing pneumatically assisted electrospray ionization. The analyte and internal standard are isolated from the plasma matrix by solid-phase extraction. The mass spectrometer is operated in the positive ion multiple reaction monitoring mode and is set to detect the presence of a precursor-product ion pair for both the analyte and internal standard to generate product ion chromatograms for both species. The analyte is quantified by using weighted least-squares regression of the peak height ratio of drug:internal standard. The method provides linear response for plasma concentrations ranging from $5 \mathrm{ng} / \mathrm{mL}$ ( $25 \mathrm{pg}$ on-column) to $2500 \mathrm{ng} / \mathrm{mL}$. Statistical evaluation and examples of authentic sample assays are also presented. (C) 1996 American Society for Mass Spectrometry (] Am Soc Mass Spectrom $1996,7,1238-1244)$
\end{abstract}

$\mathrm{T}$ There has recently been an increasing amount of attention given to fibrinogen receptor antagonists as a means of treatment and/or prevention of thrombotic and thromboembolic ischemic events such as stroke and acute myocardial infarction. These compounds exhibit antithrombotic activity by disrupting the platelet glycoprotein IIb-IIIa (GPIIbIIIa)-fibrinogen interactions-hence preventing platelet aggregation and subsequent thrombus formation. SB 214134 is a second-generation member in this class of compounds, employing a highly substituted benzodiazepine as the backbone of the molecule. To support preclinical pharmacokinetic characterization of the compound a rapid, sensitive, and specific assay was required for the quantification of SB 214134 in dog plasma. The molecule is not natively fluorescent, has a weak chromophore, is well retained on a reversed phase high-performance liquid chromatography (HPLC) system, and contains basic and acidic sites. In light of this, the use of high-performance liquid chro-

Address reprint requests to Richard C. Simpson, Department of Drug Metabolism, WP26-372, Merck Research Laboratories, P.O. Box 4, West Point, PA 19486.

- Current address: Department of Drug Metabolism, Merck Research Laboratories, West Point, PA 19486. matography-tandem mass spectrometry (HPLC/ MS/MS) was pursued as the technique having the most potential of meeting all of the assay requirements.

The literature contains numerous examples of the use of high-performance liquid chromatography-mass spectrometry (HPLC/MS) in both qualitative [1-8] and quantitative [9-33] assays. The interface and ionization techniques used in these assays have included frit flow fast-atom bombardment $[9,10]$, particle beam [11-13, 26], and thermospray [14-17], with the use of atmospheric pressure chemical ionization (APCI) $[1,18-24]$ and electrospray [2-8, 25-33] techniques becoming more widespread in some of the more recent applications. The assays have achieved mass resolution by using both single $[1,2,4-6,9-15,18-20,26-31]$ and tandem $[3,7,8,16,17,21-25,32,33]$ mass spectrometers. All of these methods have demonstrated a high degree of sensitivity or selectivity for a variety of complex matrices, ranging from environmental [27] to physiological $[1,3,4,6,9-16,18-26,28-30,32,33]$ samples.

Previous experience in our laboratory with compounds similar in structure to the present analyte indicated that the use of pneumatically assisted elec- 
trospray ionization provided more stable performance, in terms of linearity and sensitivity, than was achievable by using APCI. Due to this prior in-house experience and encouraged by the number of successful assays cited above, development of the current assay focused on the use of reversed phase microbore HPLC coupled to a triple quadrupole mass spectrometer utilizing pneumatically assisted electrospray ionization. This article describes the development and performance characterization of the resulting quantitative method.

\section{Experimental}

\section{Materials}

The analyte (SB 214134) and an analog used as the internal standard (SB 213198) were obtained from the Department of Medicinal Chemistry (SmithKline Beecham). Their structures are shown in Figure 1. HPLC-grade water (Milli-Q water purification system; Millipore Corp., Bedford, MA) was used in the preparation of all aqueous standard solutions and mobile phase. Reagent-grade formic acid was obtained from Sigma (St. Louis, $\mathrm{MO}$ ) and reagent-grade trifluoroacetic acid was supplied by Fluka (Ronkonkoma, NY). HPLC-grade acetonitrile and methylene chloride were purchased from J. T. Baker (Phillipsburg, NJ). Silica-based phenyl bonded phase solid-phase extraction cartridges $(100 \mathrm{mg} / \mathrm{mL})$ were purchased from Analytichem International (Harbor City, CA). The solid-phase extraction vacuum manifold, equipped with Teflon needles, was obtained from Supelco (Bellefonte, PA).

\section{Standards Solutions}

The stock solution of SB $214134(1 \mathrm{mg} / \mathrm{mL})$ and working standard solutions at concentrations of $100,10,1$,

a<smiles>C[C@H]1Nc2ccc(C(=O)N3CCC(C4CCNCC4)CC3)cc2CN(C)C1O</smiles>

SB 214134

b<smiles>CN(CCC1CCNCC1)C(=O)c1ccc2c(c1)N[C@H](CC(=O)O)C(=O)N(C)C2</smiles>

SB 213198

Figure 1. Structures of (a) SB 214134 and (b) SB 213198 (internal standard).
0.1 , and $0.01 \mu \mathrm{g} / \mathrm{mL}$ were prepared in $\mathrm{H}_{2} \mathrm{O}$. The aqueous stock solution of internal standard $(1 \mathrm{mg} / \mathrm{mL})$ was diluted appropriately to prepare the working internal standard solution $(4 \mu \mathrm{g} / \mathrm{mL})$. These solutions were refrigerated for storage and were stable for at least 2 months.

\section{Sample Preparation}

A $100-\mu \mathrm{L}$ aliquot of dog plasma, $10-50 \mu \mathrm{L}$ of the appropriate aqueous working standard solution of SB 214134 (to give the desired analyte concentrations if preparing calibration standards), and $50 \mu \mathrm{L}$ of working internal standard solution were briefly mixed in a $12 \times 75-\mathrm{mm}$ glass culture tube. To this was added 500 $\mu \mathrm{L}$ of an aqueous $0.1 \%$ trifluoroacetic acid solution, followed by another brief mixing. The sample was then transferred to a phenyl solid-phase extraction cartridge which had been conditioned with one column volume of methanol and one column volume of aqueous $0.1 \%$ trifluoroacetic acid. By using vacuum, the sample was passed through the cartridge and the eluate was discarded. The cartridge was then sequentially washed with $1 \mathrm{~mL}$ each of $(\mathrm{A})$ aqueous $0.1 \%$ trifluoroacetic acid, (B) aqueous $0.1 \%$ trifluoroacetic acid:acetonitrile (95:5), and (C) methylene chloride containing $0.1 \%$ trifluoroacetic acid. The isolated analyte and internal standard were eluted from the cartridge by using $1 \mathrm{~mL}$ of aqueous $0.1 \%$ trifluoroacetic acid:acetonitrile (1:1). The eluate was collected in a silanized $12 \times 75-\mathrm{mm}$ glass culture tube. The tube was placed in a water bath (approximately $45^{\circ} \mathrm{C}$ ) and the fraction was evaporated to dryness under a gentle stream of nitrogen. The resulting residue was reconstituted with $100 \mu \mathrm{L}$ of $\mathrm{H}_{2} \mathrm{O}$ and transferred to a lowvolume insert in the HPLC autosampler vial. The vial was centrifuged briefly to precipitate any suspended insoluble material.

\section{High-Performance Liquid Chromatography-Tandem Mass Spectrometry}

The HPLC system consisted of Hitachi L6200 and L6000 pumps (Danbury, CT), a model AS-3000 autosampler (Thermo Separation Products, Fremont, CA), and a SCIEX API III + mass spectrometer (PerkinElmer/Sciex, Ontario, Canada). The analytical microbore HPLC column was $50 \times 1 \mathrm{~mm}$, packed with $3-\mu \mathrm{m}$ Hypersil BDS $\mathrm{C}_{18}$ material (Keystone Scientific, Bellefonte, PA). The injection volume was set at $5 \mu \mathrm{L}$. The mobile phase consisted of aqueous $0.1 \%$ formic acid:acetonitrile (85:15) and was delivered to the column isocratically at a flow of $50 \mu \mathrm{L} / \mathrm{min}$. The mass spectrometer was operated in the multiple reaction monitoring (MRM) mode by using ion-spray ionization with an ionization potential of $5500 \mathrm{~V}$. The nebulizing gas (air) pressure was $40 \mathrm{lb} / \mathrm{in}^{2}$, the curtain gas $\left(\mathrm{N}_{2}\right)$ 
flow was $1.6 \mathrm{~L} / \mathrm{min}$, and the orifice potential was $60 \mathrm{~V}$. Ions were collisionally activated at an energy of $50 \mathrm{eV}$ and the collision gas (Ar) thickness was $125 \times 10^{13}$ atoms $/ \mathrm{cm}^{2}$. To assay for SB 214134, the mass spectrometer was operated at unit mass resolution and set to select the $[\mathrm{M}+\mathrm{H}]^{+}$ion at $\mathrm{m} / \mathrm{z} 429$ for SB 214134 and the $[\mathrm{M}+\mathrm{H}]^{+}$ion at $\mathrm{m} / \mathrm{z} 403$ for the internal standard (SB 213198) via the first quadrupole mass filter (Q1) while signals for product ions at $\mathrm{m} / \mathrm{z} 261$ for both the analyte and internal standard were monitored via the third quadrupole mass filter (Q3). The dwell time for each transition was 200 ms. Multiple reaction monitoring data was acquired by using MacSpec version 3.22 and RAD version 2.5 software and integrated by using MacQuan version 1.3 software packages (Perkin-Elmer/Sciex). Under the conditions described, SB 214134 and the internal standard were not chromatographically resolved, with each having a retention time of approximately $1.2 \mathrm{~min}$.

\section{Standard Curves}

By using the analyte and internal standard working solutions described above, a series of plasma samples were prepared having drug concentrations of $0,5,10$, $30,50,100,300,500,1000,1500,2000$, and $2500 \mathrm{ng} / \mathrm{mL}$. These samples were subjected to the solid-phase extraction procedure to generate a 12 point standard curve. The chromatographic peak height ratios of product ions for drug and internal standard (drug:internal standard) were obtained, weighted by a factor of $1 / x$ (based on analysis of residuals), and plotted versus the plasma drug concentrations. Linear regression analysis gave a calibration curve that was used to calculate plasma analyte concentrations.

\section{Precision Samples}

Precision plasma samples were prepared by using a stock analyte solution prepared from a different analyte weighing than was used to prepare standards for construction of the calibration curve. Plasma precision samples were prepared in bulk at each of six concentration levels $(5,10,20,1000,2000$, and $2500 \mathrm{ng} / \mathrm{mL}$ ). The bulk plasma samples at each level were then subdivided and aliquots were placed into individual tubes and frozen until the time of processing. Six replicates of each concentration level were processed and assayed on each of three days to obtain statistical data concerning the assay reproducibility.

\section{Results and Discussion}

\section{Compound Spectra and Resolution}

The positive ion full-scan ion-spray spectrum for the analyte and the corresponding product ion spectrum obtained from the $[\mathrm{M}+\mathrm{H}]^{+}$precursor are illustrated in Figure 2a and $b$, respectively. The corresponding
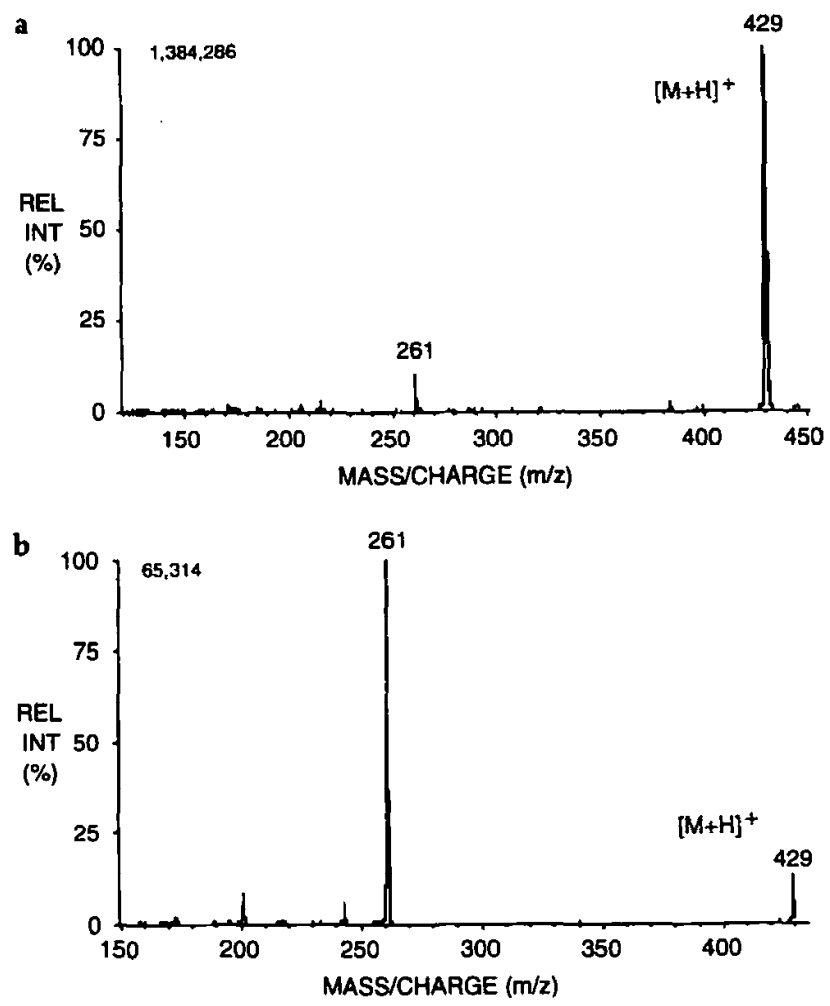

Figure 2. Positive ion ion-spray spectra of SB 214134: (a) fullscan spectrum; (b) product ion spectrum.

spectra for the internal standard are shown in Figure $3 a$ and $b$, respectively. As seen in the spectra, although the precursor ions are different for analyte and internal standard, both compounds generate the same product ion at $\mathrm{m} / \mathrm{z} 261$ produced by cleavage at the amide bond linking the benzodiazepine moiety of the molecules to the bipiperidine or piperidine components.

Under the conditions described, no chromatographic resolution is achieved between the analyte and internal standard. Both components coelute at a retention time of approximately $1.2 \mathrm{~min}$. However by utilizing the mass resolution achieved, the extracted product ion chromatograms can easily differentiate between the two components.

\section{Selectivity, Sensitivity, and Linearity}

Typical chromatograms obtained from selected monitoring of the precursor to product ion for extracts of control dog plasma and plasma spiked with internal standard only are illustrated in Figures 4 and 5, respectively. These results demonstrate the high degree of selectivity and lack of interference from any endogenous components as well as any analyte-internal standard interferences.

In any quantitative method, sensitivity is a major factor to consider, especially when dealing with limited sample sizes. The current assay employs various 

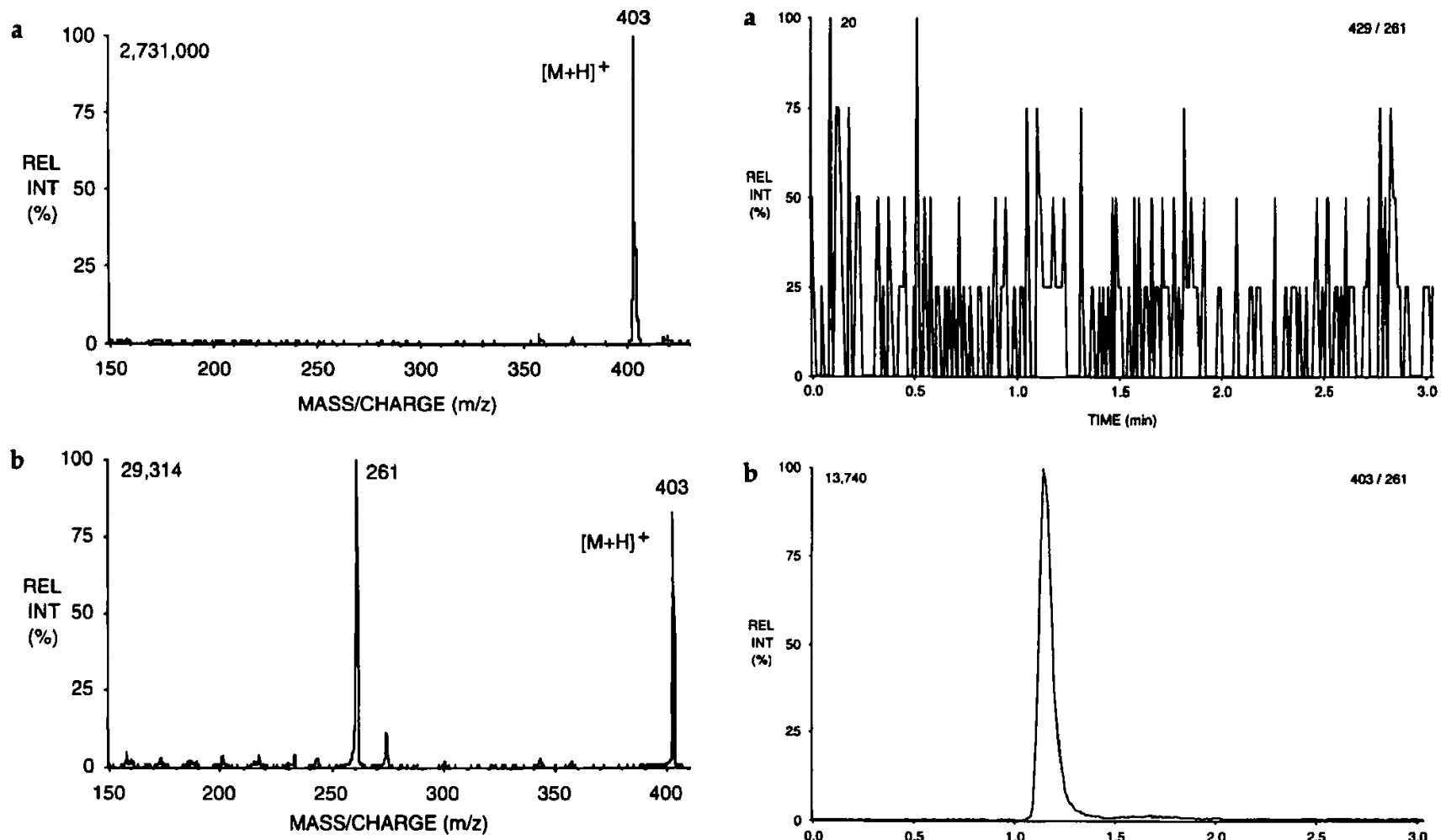

Figure 3. Positive ion ion-spray spectra of SB 213198 (internal standard): (a) full-scan spectrum; (b) product ion spectrum.

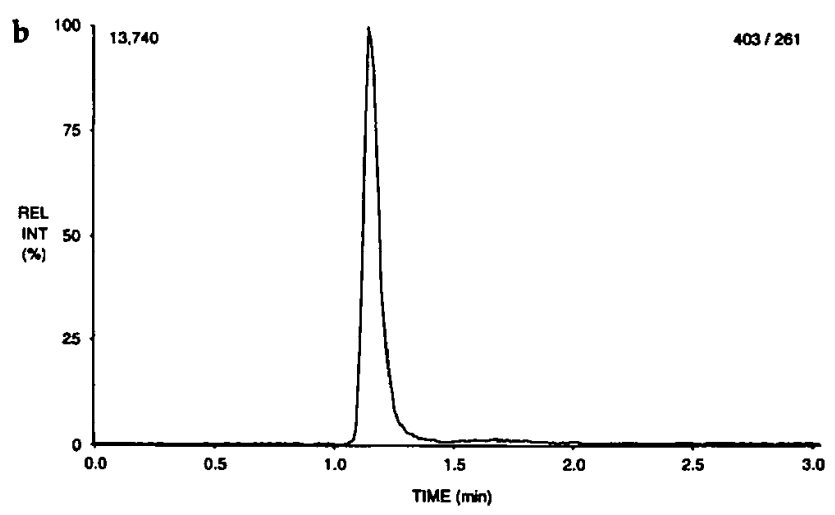

Figure 5. Chromatograms derived from selected monitoring of the precursor to product ion fragmentation for dog plasma spiked with internal standard only: (a) SB 214134; (b) SB 213198 (internal standard).
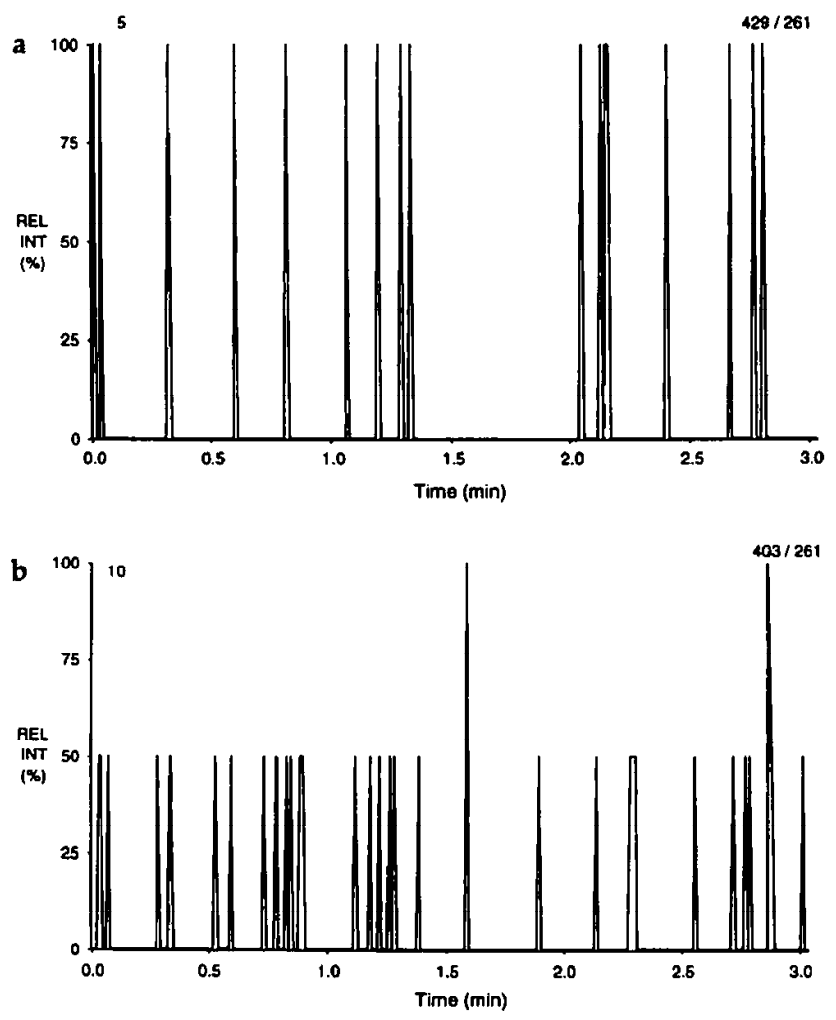

Figure 4. Chromatograms derived from selected monitoring of the precursor to product ion fragmentation for blank dog plasma: (a) SB 214134; (b) SB 213198 (internal standard).

techniques to enhance the sensitivity obtained. Many reported HPLC/MS electrospray methods utilize larger diameter HPLC columns ( $\geq 2 \mathrm{~mm}$ ) and correspondingly higher mobile phase flow rates ( $>200 \mu \mathrm{L} / \mathrm{min}$ ), necessitating the use of a postcolumn splitter to reduce the flow rate of the mobile phase entering the mass spectrometer interface to approximately $50 \mu \mathrm{L} / \mathrm{min}$ $[4-7,28-33]$ so as not to adversely impact the sensitivity. Since the electrospray interface is typically considered to act as a concentration-dependent detector, such an approach can successfully be used, provided proportionately more analyte is injected on-column as the column diameter increases. This increase is proportional to the ratio of the column diameters squared. Hence, if the column diameter is increased by a factor of 2, then the amount of analyte injected must be quadrupled to give the same detector response. However when dealing with limited-volume samples, as is often the case in preclinical metabolism studies, such an approach may not be the most effective strategy. By utilizing a small-diameter (1-mm) microbore column, as in the current method, proportionately less analyte is required to be injected on-column to achieve the same sensitivity due to the concentration-dependent behavior of the electrospray process. In addition to reduced sample consumption, no postcolumn splitter is required, since $1-\mathrm{mm}$-i.d. columns can be operated very efficiently at near-optimum performance with a 
mobile phase flow rate of $50 \mu \mathrm{L} / \mathrm{min}$. Thus all of the injected analyte is used to generate signal, with none being wasted by diversion through a postcolumn splitter such as occurs with the use of larger diameter chromatographic columns and their associated higher flow rates.

Another feature to enhance sensitivity in the current method is the use of on-column focusing of the analyte. For 1-mm-i.d. columns, especially those of short length as used here $(5 \mathrm{~cm})$, a $5-\mu \mathrm{L}$ injection volume is unusually large and would typically lead to chromatographic band distortion and/or splitting. However by reconstituting the extract residue in a very weak solvent (100\% aqueous) relative to the elution strength of the mobile phase ( $85 \%$ aqueous $/ 15 \%$ organic) larger injection volumes may be employed, with the analyte being retained and focused in a very narrow band at the column head. This is essentially on-column enrichment during the injection process, with very little migration occurring until after the injection process is completed. The result is a substantially larger analyte load than would typically be achieved, accompanied by very good chromatographic peak shape, effectively improving the sensitivity of the assay.

Utilizing the above techniques and operating the mass spectrometer in the MRM mode monitoring the analyte precursor-product ion pair of $\mathrm{m} / \mathrm{z} 429 / 261$, an on-column limit of detection (at a signal-to-noise ratio of 3) for the analyte was $2 \mathrm{ng} / \mathrm{mL}$ ( $10 \mathrm{pg}$ on-column; $23 \mathrm{fmol}$ ). Based on the described sample preparation procedure and a $5-\mu \mathrm{L}$ injection volume, linear response for the quantitative determination of analyte was obtained for plasma SB 214134 concentrations ranging from $5 \mathrm{ng} / \mathrm{mL}$ ( $25 \mathrm{pg}$ on-column) to 2500 $\mathrm{ng} / \mathrm{mL}$ (12.5 $\mathrm{ng}$ on-column). Correlation coefficients obtained by using weighted $(1 / x)$ linear regression analysis were typically 0.997 or better.

\section{Recovery}

In any quantitative assay, the recovery of the analyte should be verified across the concentration range of the assay. Quantitative recovery is not necessarily a prerequisite for a successful quantification assay, although higher analyte recovery generally translates to better assay sensitivity. However, consistent analyte recovery across the concentration range is essential to a successful assay. Inconsistent recoveries may lead to a series of problems such as nonlinear response or poor assay reproducibility. The recovery data for analyte across the assay concentration range, as well as that for the internal standard, are summarized in Table 1 . These values (mean of six replicates at each level) represent the amount of analyte determined to be present in extracted plasma samples spiked to the specific analyte concentrations relative to unextracted standards. Recovery of the analyte is consistently high (>90\%) across the range, with a slightly lower recovery observed for the internal standard. Data indicated that
Table 1. Recovery of SB 214134 and internal standard from dog plasma

\begin{tabular}{lcccc}
\hline Compound & $\begin{array}{c}\text { Conc. } \\
\text { (ng/mL) }\end{array}$ & $\begin{array}{c}\text { Mean } \\
\text { recovery } \\
(\%)\end{array}$ & $\begin{array}{c}\text { CV } \\
(\%)\end{array}$ & $N$ \\
\hline \hline SB 214134 & 5 & 94.69 & 8.27 & 6 \\
& 1000 & 98.01 & 10.70 & 6 \\
& 2500 & 90.24 & 11.61 & 6 \\
$\begin{array}{l}\text { Internal } \\
\text { standard }\end{array}$ & 2000 & 84.44 & 17.35 & 6 \\
\hline
\end{tabular}

${ }^{a}$ Coefficient of variation.

the use of silanized glassware improved analyte and internal standard recovery from the dried solid-phase extraction residue. However, the use of silanized glassware was not necessary in either the initial spiking procedure or in the autosampler vials.

\section{Assay Statistics}

Table 2 presents the statistical results obtained during a 3-day assay validation. As seen in the table, the assay performs very well across the entire concentration range, with both the within-day and between-day as-
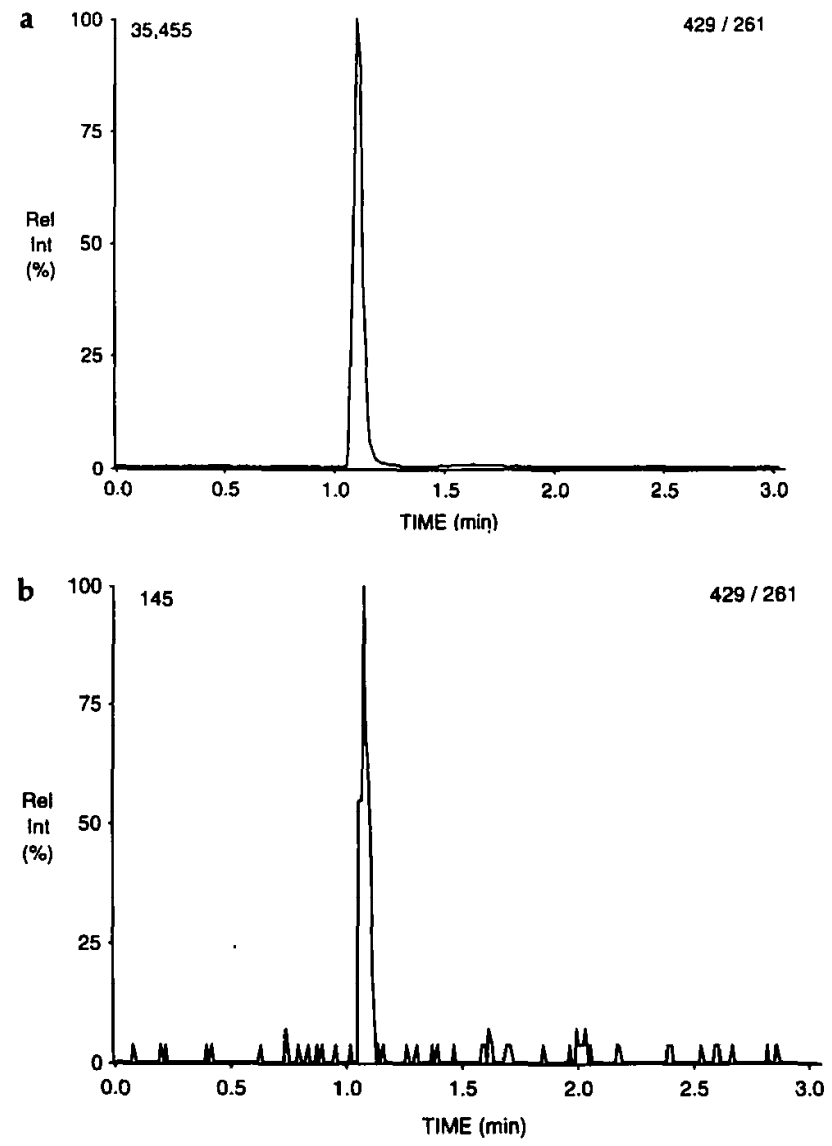

Figure 6. Chromatograms derived from selected monitoring of the precursor to product ion fragmentation for SB 214134 following IV infusion administration to the dog; (a) End of infusion $(1928 \mathrm{ng} / \mathrm{mL})$; (b) later sample time $(9 \mathrm{ng} / \mathrm{mL})$. 
Table 2. Summary of assay precision and accuracy

\begin{tabular}{|c|c|c|c|c|c|c|}
\hline $\begin{array}{l}\text { Nominal } \\
\text { conc. } \\
\text { (ng/mL) }\end{array}$ & Day 1 & Day 2 & Day 3 & $\begin{array}{c}\text { Average } \\
\text { within-run } \\
\text { precision }{ }^{a}(\%)\end{array}$ & $\begin{array}{c}\text { Between- } \\
\text { run } \\
\text { precision }^{b}(\%)\end{array}$ & $\begin{array}{c}\text { Average } \\
\text { accuracy }^{c}(\%)\end{array}$ \\
\hline 5 & & & & 8.68 & 4.73 & 101.0 \\
\hline Mean & 4.79 & 5.12 & 5.25 & & & \\
\hline Std. dev. & 0.46 & 0.49 & 0.36 & & & \\
\hline$\% \mathrm{CV}$ & 9.61 & 9.50 & 6.93 & & & \\
\hline$\%$ Accy. & 95.7 & 102.3 & 105.0 & & & \\
\hline 10 & & & & 9.25 & 1.31 & 98.8 \\
\hline Mean & 9.79 & 9.83 & 10.03 & & & \\
\hline Std. dev. & 0.95 & 0.91 & 0.89 & & & \\
\hline$\% \mathrm{CV}$ & 9.69 & 9.23 & 8.84 & & & \\
\hline$\%$ Accy. & 97.9 & 98.3 & 100.3 & & & \\
\hline 20 & & & & 8.57 & 2.57 & 97.8 \\
\hline Mean & 20.06 & 19.06 & 19.53 & & & \\
\hline Std. dev. & 1.67 & 1.46 & 1.90 & & & \\
\hline$\% \mathrm{CV}$ & 8.31 & 7.68 & 9.71 & & & \\
\hline$\%$ Accy. & 100.3 & 95.3 & 97.6 & & & \\
\hline 1000 & & & & 6.35 & 4.89 & 100.7 \\
\hline Mean & 965.56 & 1061.54 & 994.30 & & & \\
\hline Std. dev. & 66.00 & 35.98 & 87.87 & & & \\
\hline$\% \mathrm{CV}$ & 6.84 & 3.39 & 8.84 & & & \\
\hline$\%$ Accy. & 96.6 & 106.2 & 99.4 & & & \\
\hline 2000 & & & & 7.44 & 3.85 & 98.9 \\
\hline Mean & 2054.70 & 1902.56 & 1975.89 & & & \\
\hline Std. dev. & 126.99 & 167.03 & 145.34 & & & \\
\hline$\% \mathrm{CV}$ & 6.18 & 8.78 & 7.36 & & & \\
\hline$\%$ Accy. & 102.7 & 95.1 & 98.8 & & & \\
\hline 2500 & & & & 4.05 & 1.45 & 101.1 \\
\hline Mean & 2489.70 & 2563.08 & 2526.25 & & & \\
\hline Std. dev. & 110.05 & 100.53 & 96.40 & & & \\
\hline$\% \mathrm{CV}$ & 4.42 & 3.92 & 3.82 & & & \\
\hline$\%$ Accy. & 99.6 & 102.5 & 101.1 & & & \\
\hline
\end{tabular}

Average within-run precision $(\%)=\left(C V_{\text {run } 1}+C V_{\text {run } 2}+C V_{\text {run } 3}\right) /$ number of runs.

Between-run precision $(\%)=$ (std. dev. of within-day means $\times 100)$ /average within-day mean.

${ }^{c}$ Average accuracy $(\%)=$ (accuracy $_{\text {run } 1}+$ accuracy $_{\text {run } 2}+$ accuracy $\left._{\text {run } 3}\right) /$ number of runs.

say precision $\leq 10 \%$ in all cases. The accuracy is also acceptable across the concentration range, varying from 97.8 to $101.1 \%$. The use of peak height ratios rather than peak area ratios resulted in marginally better statistical performance.

\section{Authentic Examples}

The described method has been applied to dog plasma samples for quantification of the analyte following administration of the compound via various dosing regimens. Figure 6a shows the chromatogram obtained from selected monitoring of the analyte precursor to product ion for an extract of a plasma sample obtained immediately following an intravenous infusion administration of the drug to a dog. The plasma drug concentration in this sample was determined to be nearly $2000 \mathrm{ng} / \mathrm{mL}$. Figure $6 \mathrm{~b}$ shows the chromatogram obtained from selected monitoring of the analyte precursor to product ion obtained for the extract of a plasma sample obtained from the same dog at a significantly later time point. The plasma analyte concentration for this sample was still quantifiable and determined to be less than $10 \mathrm{ng} / \mathrm{mL}$. These two figures demonstrate the utility of a method having both a wide linear response range as well as adequate sensitivity. This method has been successfully used to generate plasma drug concentration versus time profiles of the analyte following a variety of dosing regimens, with each study consisting of approximately 100-150 samples, allowing for the rapid generation of quantitative data used for the subsequent pharmacokinetic characterization of the drug's performance in the dog model.

\section{Conclusions}

In conclusion, the assay described is rapid, with chromatographic elution of the analyte and internal standard complete in less than $2 \mathrm{~min}$. The assay is selec- 
tive, with no evidence of any interferences. The linear response range for quantification is from $5 \mathrm{ng} / \mathrm{mL}(25$ $\mathrm{pg}$ on-column) to $2500 \mathrm{ng} / \mathrm{mL}$ (12.5 $\mathrm{ng}$ on-column), with a limit of detection of $2 \mathrm{ng} / \mathrm{mL}$ (10 pg oncolumn). The assay is based on easily obtainable sample volumes, and statistical evaluation proved the method to be reliable over the concentration range described. Finally, the method has successfully been applied to preclinical pharmacokinetic studies.

\section{References}

1. Kasuya, F.; Igarashi, K.; Fukui, M. J. Chromatogr. A 1994, 684, 93-101.

2. Galceran, M. T.; Moyano, E. J. Chromatogr. A 1994, 683, 9-19.

3. Poon, G. K.; Raynaud, F. I.; Mistry, P.; Odell, D. E.; Kelland, L. R.; Harrap, K. R.; Barnard, C. F. J.; Murrer, B. A. J. Chromatogr. A 1995, 712, 61-66.

4. Jones, R. M.; Yuan, Z.-X.; Lamb, J. H.; Lim, C. K. J. Chromatogr. A 1996, 722, 249-255.

5. Bleicher, K.; Bayer, E. Chromatographia 1994, 39, 405-408.

6. Roda, A.; Gioacchini, A. M.; Cerre, C.; Baraldini, M. 1. Chromatogr. B 1995, 665, 281-294.

7. Jones, R. M.; Wang, Q.; Lamb, J. H.; Djela, B. D.; Lim, C. K. J. Chromatogr. A 1996, 722, 257-265.

8. Qin, X.-Z.; Tsai, E. W.; Sakuma, T.; Ip, D. P. J. Chromatogr. A 1994, 686, 205-212.

9. Hieda, Y.; Kashimura, S.; Hara, K.; Kageura, M. J. Chromatogr. A 1995, 667, 241-246.

10. Matsui, K.; Oda, Y.; Ohe, H.; Tanaka, S.; Asakawa, N. J. Chromatogr. A 1995, 667, 209-218.

11. Stanley, S. M. R.; Wilhelmi, B. S.; Rodgers, J. P.; Guthrie, A. Biol. Mass Spectrom. 1994, 23, 483-491.

12. Girault, J.; Longueville, D.; Malgouyat, J. M.; Istin, B.; Lecomte, G.; Fourtillan, J. B. Chromatographia 1994, 39, 228-238.

13. Girault, J.; Malgouyat, J. M.; Longueville, D.; Lecomte, G.; Revaud, M.; Fourtillan, J. B. J. Chromatogr. B 1994, 658, 289-301.
14. Valeur, A.; Olsson, N. U.; Kaufmann, P.; Wada, S.; Kroon, C.-G.; Westerdahl, G.; Odham, G. Biol. Mass Spectrom. 1994, 23, 313-319.

15. Skarping, G.; Dalene, M.; Brunmark, P. Chromatographia 1994, $39,619-623$.

16. Verweij, A. M. A.; Hordijk, M. L.; Lipman, P. J. L. J. Liq. Chromatogr. 1994, 17, 4099-4110.

17. Eckers, C.; Hutton, K. A.; de Biassi, V.; East, P. B.; Haskins, N. J.; Jacewicz, V. W. J. Chromatogr. A 1994, 686, 213-218.

18. Yoshida, K.; Kondo, F. J. Liq. Chromatogr. 1994, 17, 2625-2632.

19. Herron, W. J.; Eadie, J.; Penman, A. D. J. Chromatogr. A 1995, $712,55-60$.

20. Jianying, Z.; Machida, Y.; Sugahara, K.; Kodama, H. J. Chromatogr. B 1994, 660, 375-379.

21. Olah, T. V.; Gilbert, J. D.; Barrish, A.; Greber, T. F.; McLoughlin, D. A. I. Pharm. Biomed. Anal. 1994, 12, 705-712.

22. Constanzer, M. L.; Chavez, C. M.; Matuszewski, B. K. J. Chromatogr. B 1994, 658, 281-287.

23. Constanzer, M.; Chavez, C.; Matuszewski, B. J. Chromatogr. B $1995,666,117-126$.

24. Murphy, A. T.; Kasper, S. C.; Gillespie, T. A.; Delong, A. F. J. Chromatogr. B 1995, 668, 273-280.

25. Lausecker, B.; Hopfgartner, G. I. Chromatogr. A 1995, 712, 75-83.

26. Stanley, S. M. R.; Owens, N. A.; Rodgers, J. P. I. Chromatogr. B 1995, 667, 95-103.

27. Chiron, S.; Papilloud, S.; Haerdi, W.; Barcelo, D. Anal. Chem. 1995, 67, 1637-1643.

28. Hayes, F. J.; Baker, T. R.; Dobson, R. L. M.; Tsueda, M. S. J. Chromatogr. B 1995, 692, 73-81.

29. Pacifici, R.; Pichini, S.; Altieri, I.; Caronna, A.; Passa, A. R.; Zuccaro, P. J. Chromatogr. B 1995, 664, 329-334.

30. Mutlib, A. E.; Strupczewski, J. T. I. Chromatogr. B 1995, 669, 237-246.

31. Bryant, D. K.; Kingswood, M. D.; Belengeur, A. J. Chromatogr A 1995, 721, 41-51.

32. Knebel, N. G.; Sharp, S. R.; Madigan, M. J. J. Chromatogr. B $1995,673,213-222$.

33. Muck, W. M. J. Chromatogr. A 1995, 712, 45-53. 